TITLE:

\title{
Vesicular GABA Uptake Can Be Rate Limiting for Recovery of IPSCs from Synaptic Depression
}

\section{AUTHOR(S):}

Yamashita, Manami; Kawaguchi, Shin-ya; Hori, Tetsuya; Takahashi, Tomoyuki

\section{CITATION:}

Yamashita, Manami ... [et al]. Vesicular GABA Uptake Can Be Rate Limiting for Recovery of IPSCs from Synaptic Depression. Cell reports 2018, 22(12): 3134-3141

\section{ISSUE DATE:}

2018-03-20

URL:

http://hdl.handle.net/2433/230367

\section{RIGHT:}

(C) 2018 The Authors. This is an open access article under the CC BY-NCND license (http://creativecommons.org/licenses/by-nc-nd/4.0/). 


\section{Reports}

\section{Vesicular GABA Uptake Can Be Rate Limiting for Recovery of IPSCs from Synaptic Depression}

\section{Graphical Abstract}

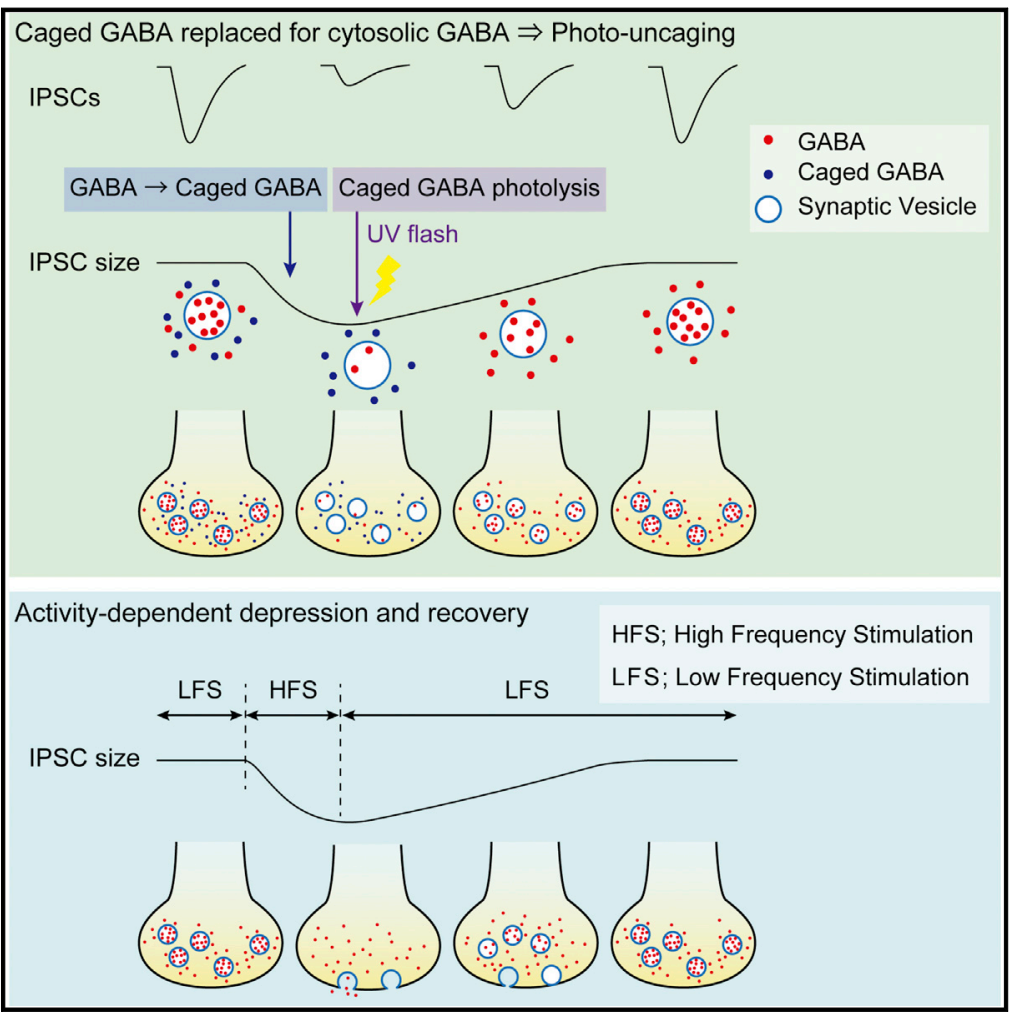

Highlights

- Vesicular GABA uptake time constant is $40 \mathrm{~s}$ at physiological temperature

- Recovery rate of IPSCs from depression coincides with vesicular GABA uptake rate

- Vesicular GABA uptake can be rate limiting for recovery of IPSCs from depression

\section{Authors}

Manami Yamashita, Shin-ya Kawaguchi, Tetsuya Hori, Tomoyuki Takahashi

\section{Correspondence}

thori@mail.doshisha.ac.jp (T.H.), ttakahas@oist.jp (T.T.)

\section{In Brief}

Recovery of inhibitory synaptic transmission from activity-dependent depression requires refilling of vesicles with GABA. Yamashita et al. find that vesicular uptake rate of GABA is a slow process, limiting the recovery rate of IPSCs from depression. 


\title{
Vesicular GABA Uptake Can Be Rate Limiting for Recovery of IPSCs from Synaptic Depression
}

\author{
Manami Yamashita, ${ }^{1,2}$ Shin-ya Kawaguchi, ${ }^{3}$ Tetsuya Hori, ${ }^{4, *}$ and Tomoyuki Takahashi ${ }^{5,6, *}$ \\ ${ }^{1}$ Laboratory of Molecular Synaptic Function, Graduate School of Brain Science, Doshisha University, Kyoto 610-0394, Japan \\ 2Department of Physiology, Faculty of Medicine, Osaka Medical College, Osaka 569-8686, Japan \\ ${ }^{3}$ Society-Academia Collaboration for Innovation, Kyoto University, Kyoto 606-8501, Japan \\ ${ }^{4}$ Department of Neurophysiology, Graduate School of Life and Medical Sciences, Doshisha University, Kyoto 610-0394, Japan \\ ${ }^{5}$ Cellular and Molecular Synaptic Function Unit, Okinawa Institute of Science and Technology (OIST) Graduate University, \\ Okinawa 904-0495, Japan \\ 6 Lead Contact \\ *Correspondence: thori@mail.doshisha.ac.jp (T.H.), ttakahas@oist.jp (T.T.) \\ https://doi.org/10.1016/j.celrep.2018.02.080
}

\section{SUMMARY}

Synaptic efficacy plays crucial roles in neuronal circuit operation and synaptic plasticity. Presynaptic determinants of synaptic efficacy are neurotransmitter content in synaptic vesicles and the number of vesicles undergoing exocytosis at a time. Bursts of presynaptic firings depress synaptic efficacy, mainly due to depletion of releasable vesicles, whereas recovery from strong depression is initiated by endocytic vesicle retrieval followed by refilling of vesicles with neurotransmitter. We washed out presynaptic cytosolic GABA to induce a rundown of IPSCs at cerebellar inhibitory cell pairs in slices from rats and then allowed fast recovery by elevating GABA concentration using photo-uncaging. The time course of this recovery coincided with that of IPSCs from activity-dependent depression induced by a train of high-frequency stimulation. We conclude that vesicular GABA uptake can be a limiting step for the recovery of inhibitory neurotransmission from synaptic depression.

\section{INTRODUCTION}

At chemical synapses, neurotransmitters are stored in synaptic vesicles (SVs) and released by exocytosis. After exocytosis, SVs are retrieved by endocytosis, refilled with neurotransmitters, and recycled to be reused for a subsequent round of transmission (Heuser and Reese, 1973; Rizzoli, 2014). Vesicular uptake of glutamate (Maycox et al., 1988; Carlson et al., 1989) or GABA (Kish et al., 1989; Hell et al., 1990) takes 5-10 min in isolated vesicles. However, glutamate uptake measured at the calyx of Held presynaptic terminal, using caged glutamate, revealed a much faster time constant of $7 \mathrm{~s}$ at physiological temperature (PT) (Hori and Takahashi, 2012). This estimation was made from the recovery rate of excitatory postsynaptic currents (EPSCs) after glutamate uncaging, based on the fact that vesicular transmitter content is in dynamic equilibrium with its cytosolic concentration (Ishikawa et al., 2002; Yamashita et al.,
2009). This uncaging method established at giant presynaptic terminals is widely applicable to neuronal pairs, where the presynaptic endogenous neurotransmitter can be replaced by caged transmitter compounds. At brainstem inhibitory neuronal pairs, the recovery time of inhibitory postsynaptic currents (IPSCs) was measured using caged glutamate photolysis (Apostolides and Trussell, 2013). This recovery time comprises both the time for GABA synthesis from glutamate by glutamate decarboxylase (GAD) and the time of GABA uptake into vesicles. Since the rate of GABA synthesis from cytosolic glutamate is undetermined, the vesicular GABA uptake rate remains unknown. Hence, in cerebellar basket cell (BC)-Purkinje cell (PC) pairs, we directly measured vesicular GABA uptake rate using caged GABA. Our results indicated that the vesicular GABA uptake rate is $5-6$ times slower than the glutamate uptake rate (Hori and Takahashi, 2012).

Given that the vesicular GABA uptake rate is slow, it might limit the recovery rate of IPSCs from short-term depression (STD). During high-frequency stimulations, IPSCs undergo STD, mainly due to the depletion of releasable SVs. Recovery of neurotransmission from STD is initiated by the endocytic retrieval of SVs, followed by their refilling with neurotransmitter and transport to release sites. At excitatory synapses, vesicular transmitter refilling is not considered as rate limiting for the recovery of neurotransmission from STD (e.g., Zucker and Regehr, 2002). In fact, vesicular glutamate uptake (Hori and Takahashi, 2012) is faster than the recovery of EPSC amplitudes after strong STD (Liu and Tsien, 1995; Ryan et al., 1993). However, at GABAergic inhibitory synapses, we found here that the recovery rate of IPSCs from STD is close to the vesicular GABA uptake rate. Furthermore, the rates of GABA uptake and recovery of IPSCs from STD showed a similar dependence on presynaptic cytosolic GABA concentrations. Thus, at inhibitory synapses, the vesicular GABA uptake rate can serve as the rate-limiting step for recovery of neurotransmission from STD.

\section{RESULTS}

IPSC Rundown by Presynaptic Cytosolic GABA Washout and Its Recovery by GABA Loading

In whole-cell pair recordings from a BC and a PC in cerebellar slices of rats, we evoked IPSCs by a train of 5 action potentials 
A

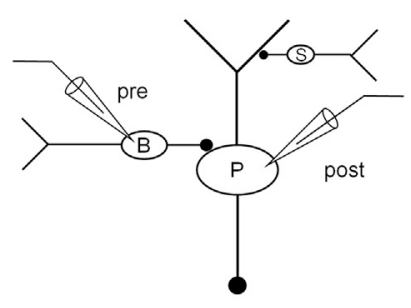

\section{C}
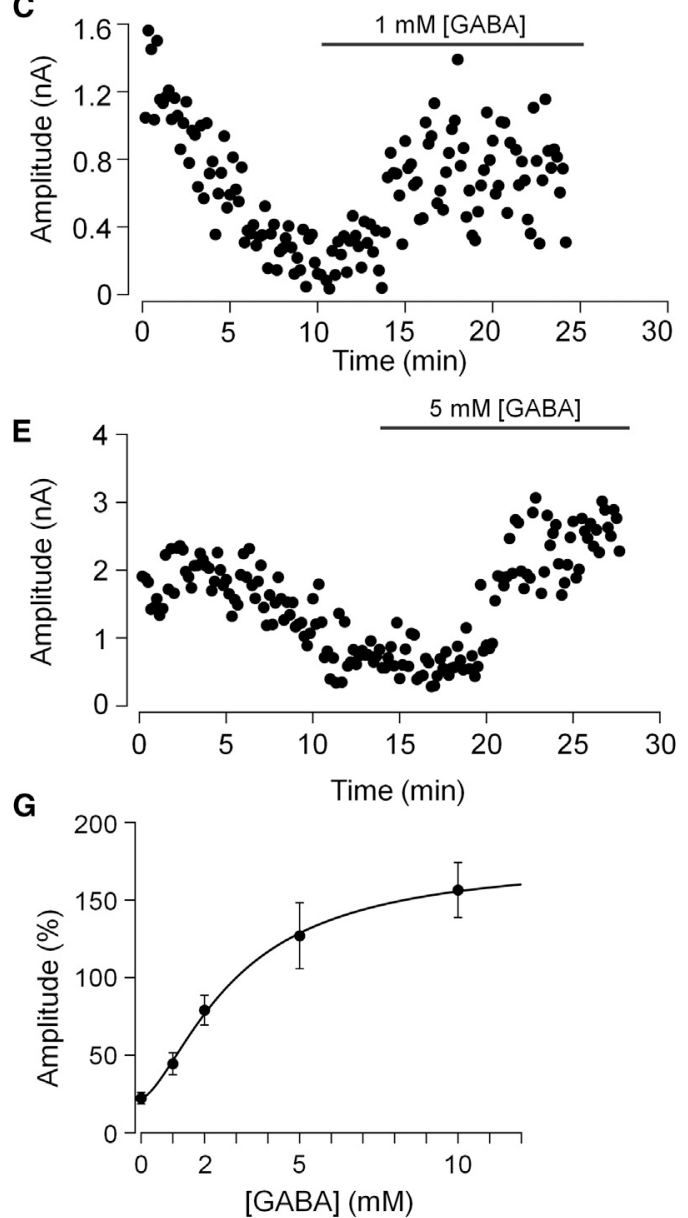
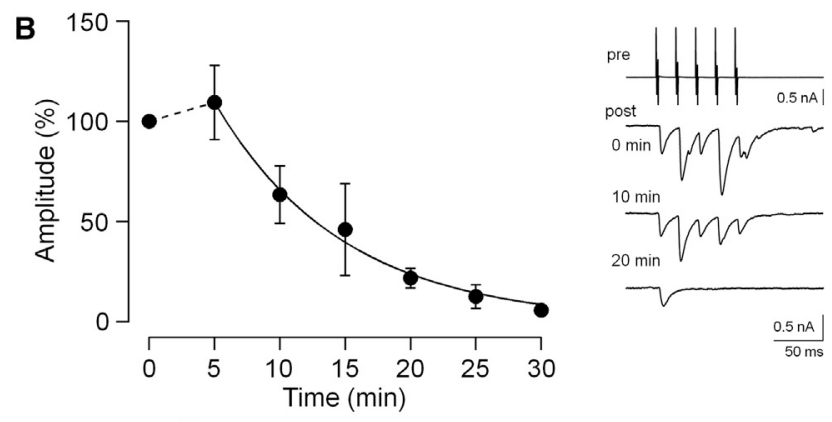

D
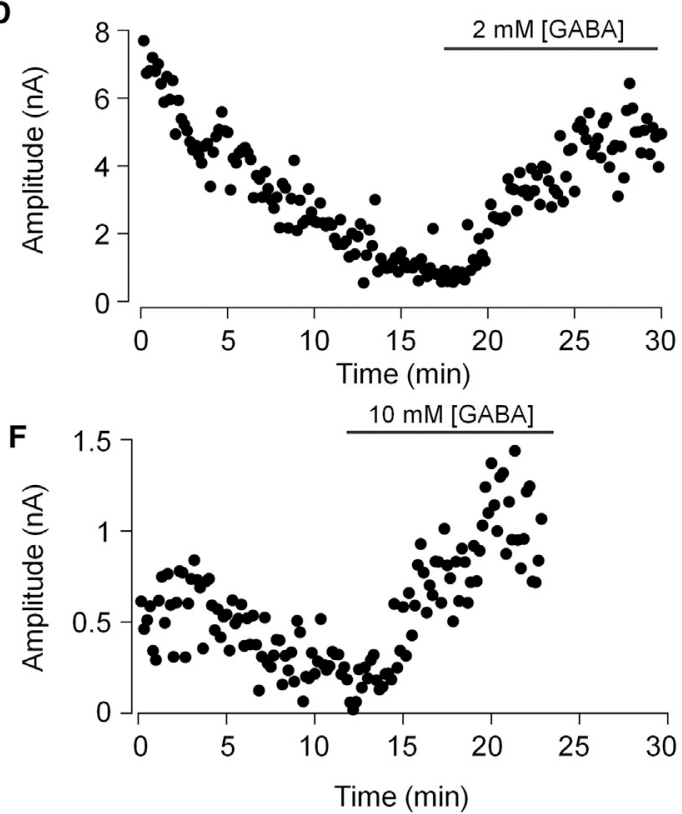

Figure 1. IPSC Rundown after GABA Washout and Recovery after GABA Reloading

(A) Schematic illustration of a pair recording from a presynaptic BC (B) and a postsynaptic PC (P). S, stellate cell.

(B) Mean amplitude of IPSCs recorded from a PC after whole-cell washout of cytosolic GABA in a BC, normalized to IPSC amplitude at time 0 . Sample traces on the right show IPSCs evoked by a train of 5 stimuli at $50 \mathrm{~Hz}$, at different time periods after membrane rupture with a whole-cell pipette. Data points and error bars indicate means and SEM of IPSC amplitude $(n=4$ pairs). Experiments were performed at RT.

(C-F) Amplitudes of IPSCs evoked at $0.1 \mathrm{~Hz}$ by presynaptic APs, measured from 3 to $4 \mathrm{~min}$ after whole-cell rupture. After IPSCs ran down to a low steady level, GABA was infused into a presynaptic $B C$ via pipette perfusion: $n=5$ at $1 \mathrm{mM}(C), n=5$ at $2 \mathrm{mM}(\mathrm{D}), \mathrm{n}=6$ at $5 \mathrm{mM}(\mathrm{E})$, and $\mathrm{n}=5$ at $10 \mathrm{mM}(\mathrm{F})$. Experiments were performed at PT.

(G) Relationship between presynaptic cytosolic GABA concentrations ([GABA]) and percentage of recovery of IPSCs (IPSC amplitude after GABA loading relative to that before washout). The curve fit represents the Hill equation

$$
\text { IPSC amplitude }=\text { IPSC amplitude after GABA washout }+(\text { maximal magnitude of potentiation }) \times \frac{[G A B A]^{n}}{[G A B A]^{n}+\left(E C_{50}\right)^{n}},
$$

where $\mathrm{n}$ and EC50 represents the Hill coefficient (1.5) and 50\% effective concentration of GABA (3.0 mM), respectively. The curve fit was constrained at $0 \mathrm{mM}[\mathrm{GABA}]$ determined from the mean IPSC amplitude 1 min before GABA loading. Maximal IPSC amplitude was determined 
A

$$
0 \mathrm{mM} \text { [GABA] } \quad 5 \mathrm{mM}[\mathrm{GABA}]
$$

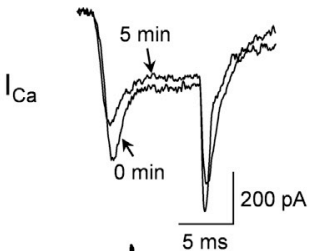

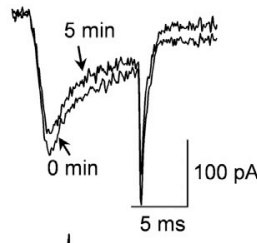

PSCs

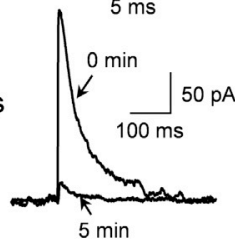

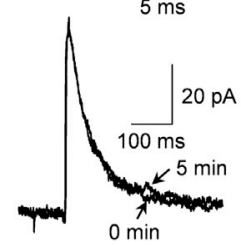

C

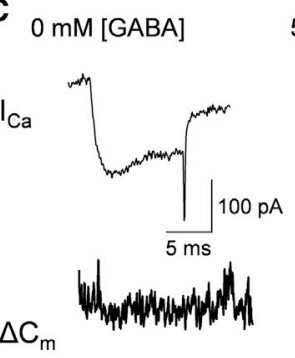

偊

$$
\underset{200 \mathrm{~ms}}{\stackrel{10 \mathrm{fF}}{2}}
$$

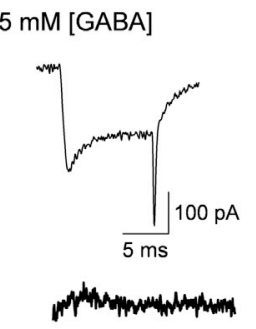

D
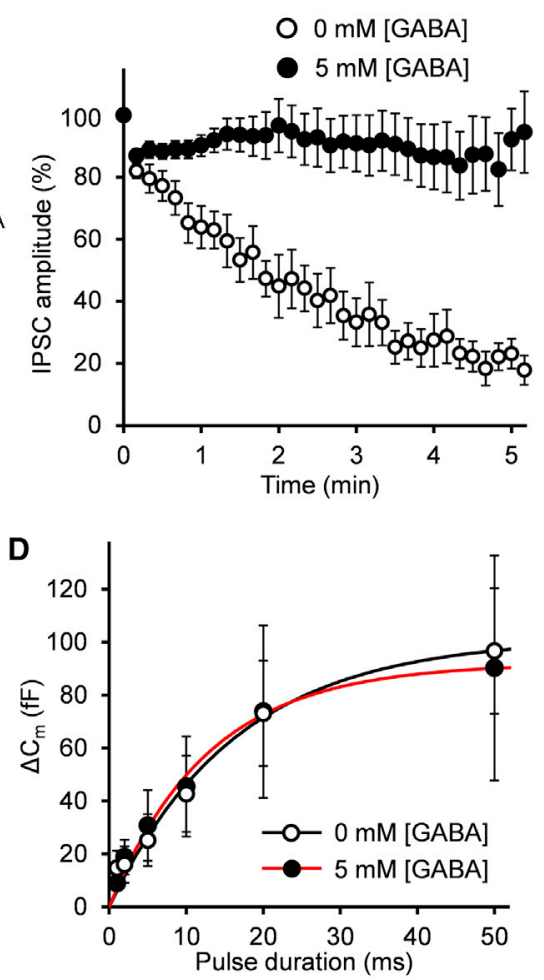

Figure 2. Cytosolic GABA Concentrations Do Not Influence the Number of Vesicles Undergoing Exocytosis at PC Axon Terminals in Culture

(A) Presynaptic $\mathrm{Ca}^{2+}$ currents $\left(\mathrm{I}_{\mathrm{Ca}}\right)$ evoked by 10-ms depolarizing pulses to $0 \mathrm{mV}$ (upper traces) and IPSCs (lower traces) simultaneously recorded from a PC terminal and a neuron from the DCN respectively, without $(0 \mathrm{mM})$ or with $(5 \mathrm{mM}) \mathrm{GABA}$ in the presynaptic pipette at RT.

(B) Time plots of relative IPSC amplitude (normalized to values at time 0 ), with (filled black circles) or without (open black circles) GABA in the presynaptic pipettes ( $n=7$ pairs for both data). At 5 min after whole-cell dialysis, IPSC amplitudes, with or without GABA in pipette solutions, were significantly different ( $p=0.0002$, Student's t test).

(C) Presynaptic membrane capacitance changes $\left(\mathrm{C}_{\mathrm{m}}\right)$ in response to $\mathrm{I}_{\mathrm{Ca}}$ elicited by a depolarizing command pulse (to $0 \mathrm{mV}$ for $10 \mathrm{~ms}$ ) at PT $\left(32^{\circ} \mathrm{C}-35^{\circ} \mathrm{C}\right)$.

(D) $\triangle \mathrm{Cm}$ induced by command pulses of different durations $(1-50 \mathrm{~ms})$ with presynaptic pipettes containing $0 \mathrm{mM}$ or $5 \mathrm{mM}$ GABA. There was no significant difference in the magnitude of $\Delta \mathrm{C}_{\mathrm{m}}$ between two groups, with or without GABA ( 6 pairs for each; $p=0.99$, repeated-measures ANOVA). Values are given as mean \pm SEM.
(APs) every 5 min (Figures $1 \mathrm{~A}$ and $1 \mathrm{~B}$ ). When the presynaptic pipette contained neither GABA nor glutamate, IPSCs underwent a rundown, with an exponential time constant of $9.8 \pm$ $1.2 \mathrm{~min}$ at room temperature $(\mathrm{RT})(\mathrm{n}=4$; Figure $1 \mathrm{~B})$, suggesting a passive leak of GABA from vesicles. We also blocked vesicular GABA uptake using a vacuolar ATPase blocker, bafilomycin A1 (Baf). After bath application of Baf $(5 \mu \mathrm{M})$, IPSCs ran down with a time constant of $11.4 \pm 2.3 \mathrm{~min}(n=5$; Figure $S 1 \mathrm{~A})$ like that after GABA washout (Figure 1B; $p=0.57$, Student's $t$ test). The IPSC rundown was faster during higher frequency stimulation (Figure S1A), suggesting an additional contribution of empty vesicles accumulated by recycling (Takami et al., 2017). The blocking effect of Baf was irreversible (Figure S1B), confirming that vesicular transmitter refilling is indispensable for recovery from depression.

Since rundown of EPSCs by glutamate washout is rescued by loading glutamate into the presynaptic cytosol (Hori and Takahashi, 2012), we likewise infused GABA into BCs using patch pipette perfusion (Hori et al., 1999) at different concentrations (1-10 mM) to let them recover (Figures 1C-1G). Full recovery of IPSCs was observed with GABA above $5 \mathrm{mM}$ (Figure 1G), suggesting that endogenous GABA concentrations in BCs are $\sim 5 \mathrm{mM}$, as in other inhibitory neurons (Apostolides and Trussell,
2013; Otsuka et al., 1971). After a 10-mM GABA infusion, recovery of IPSCs significantly exceeded the initial control level (Figure $1 \mathrm{G}$ ), suggesting that postsynaptic GABA receptors are not saturated by vesicular GABA, as at glutamatergic calyceal synapses (Ishikawa et al., 2002).

Although GABA-concentration-dependent rundown and recovery of IPSCs are likely caused by changes in vesicular GABA content, they are also proposed to result from changes in the number of releasable vesicles at hippocampal inhibitory synapses in culture (Wang et al., 2013). We tested the latter possibility using a direct whole-cell recording from PC axon terminals in culture (Kawaguchi and Sakaba, 2015). In paired recordings from a PC axon terminal and a cell from the deep cerebellar nuclei (DCN), presynaptic GABA washout caused a rundown of IPSCs (Figures 2A and 2B), as at BC-PC or other inhibitory synapses. Presynaptic membrane capacitance changes were then evoked by depolarizing pulses in the presence of GABA $(5 \mathrm{mM})$ or in its absence (Figures $2 \mathrm{C}$ and 2D). No significant difference was found between them (Figure 2D; $p=0.99$, repeated-measures ANOVA), indicating that presynaptic cytosolic transmitter concentration has no effect on SV availability at inhibitory synapses as at excitatory synapses (Hori and Takahashi, 2012; Takami et al., 2017).

from the mean IPSC amplitude 5-10 min after GABA loading. Compared with control before GABA infusion, mean IPSC amplitudes were significantly smaller at $1 \mathrm{mM}$ [GABA] ( $p=0.0014$, paired t test) and significantly larger at $10 \mathrm{mM}[\mathrm{GABA}](\mathrm{p}=0.033$, paired $t$ test). In a non-parametric Steel-Dwass test, there was also a significant difference in IPSC amplitudes between $1 \mathrm{mM}$ and $5 \mathrm{mM}[\mathrm{GABA}](p=0.04)$. Values are given as mean \pm SEM. 
A

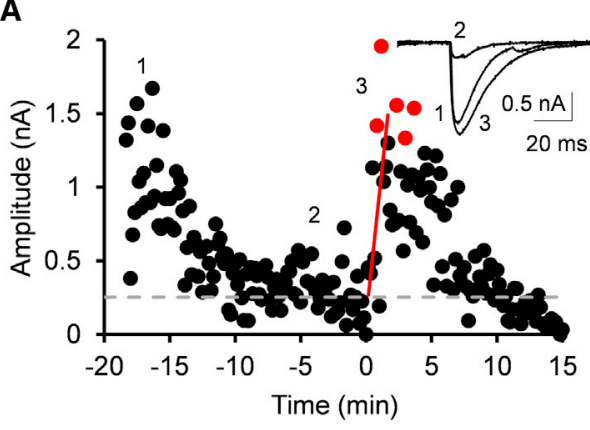

C

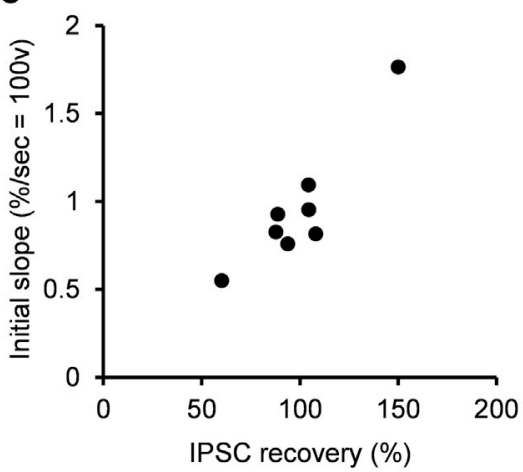

E

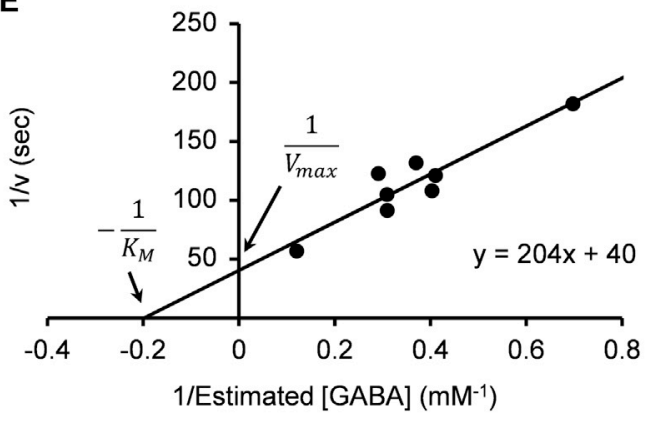

B

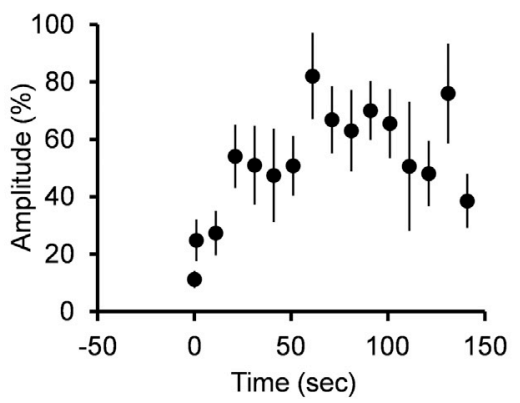

D

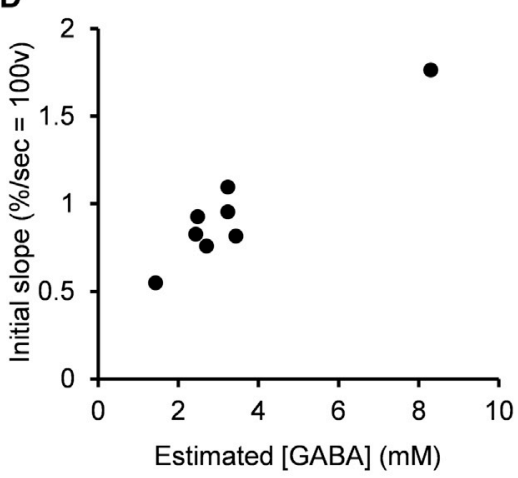

Figure 3. Estimation of Vesicular GABA Uptake Rate Using GABA Uncaging

(A) Recovery of IPSCs from rundown by UV pulse (1 s) that uncaged DPNI-GABA loaded at $10 \mathrm{mM}$. Sample records in insets show IPSCs before GABA washout (1) and after rundown of IPSCs (2 and 3) before (2) and after (3) GABA uncaging (superimposed). Data points between $1 \mathrm{~s}$ and $100 \mathrm{~s}$ after GABA uncaging were fit by regression lines with the least-squares method (red lines) and extended to the peak of recovery (red circles). The gray dashed line indicates baseline. Black circles and red circles indicate amplitude of IPSCs evoked at $0.1 \mathrm{~Hz}$. Experiments were performed at PT.

(B) Summary data from 8 cell pairs showing means and SEM of IPSC amplitude (percent recovery) after GABA uncaging.

(C) The initial slope of IPSC recovery after GABA uncaging (ordinate) plotted against the percentage of recovery of IPSCs measured from the peak of recovery (abscissa).

(D) The initial slope of IPSC recovery after GABA uncaging (ordinate) plotted against cytosolic GABA concentrations estimated from percentage of IPSC recovery after GABA infusion in Figure $1 \mathrm{G}$. (E) Lineweaver-Burk plot for the inverted uptake rate $(1 / \mathrm{v})$ versus inverted cytosolic GABA concentrations (1/[GABA]). $V_{\max }$ estimated from the ordinate intercept corresponds to a 40-s time constant, and $\mathrm{K}_{\mathrm{M}}$ from the abscissa intercept indicates $5.0 \mathrm{mM}$.

At BC-PC synapses, in the presence of $10 \mathrm{mM}$ GABA and $20 \mathrm{mM}$ glutathione in the presynaptic pipettes, UV uncaging of DPNI-GABA (10 mM) had no significant effect on the IPSC amplitude ( $p=0.94$, paired t test; Figure S2, $n=4$ ). At BC-PC synapses, immediately after presynaptic GABA uncaging, IPSC amplitude started to recover and then reached a peak, but

The Rate of GABA Uptake into Vesicles

Since cytosolic GABA concentration determines IPSC amplitude, primarily through vesicular GABA contents (Figures 1 and 2), we estimated the rate of GABA uptake into SVs from the recovery time of IPSCs from rundown. Whole-cell infusion of GABA at a given concentration provides a reliable measure for the magnitude of IPSC recovery (Figures 1C-1G), but its rate includes the diffusion time of GABA from pipettes to $B C$ terminals. To obtain a more reliable measure for the GABA uptake rate, we replaced GABA with 1-(4-Aminobutanoyl)-4-[1,3-bis(dihydroxyphosphoryloxy)propan-2-yloxy]-7-nitroindoline (DPNI)caged GABA (10 mM) in the pipette solution and let IPSCs run down. When IPSCs reached a minimal level, we applied a UV flash (1 s) through an objective lens, thereby photo-releasing GABA from caged GABA within a BC (Figure 3). UV photolysis of caged compounds in presynaptic terminals often induces toxic effects, presumably due to free radicals arising from uncaging. These effects can, however, be minimized by including glutathione in presynaptic pipettes (Hori and Takahashi, 2012).

it was soon followed by a decline (Figures $3 \mathrm{~A}$ and $3 \mathrm{~B}$ ), presumably because a bulk of DPNI-GABA remaining in the whole-cell pipette washed out newly uncaged GABA. Since the time constant of IPSC recovery after GABA uncaging $(1.9 \pm 0.2 \mathrm{~min}$, $\mathrm{n}=8$; Figure $3 \mathrm{~B}$ ) was much faster than that of IPSC rundown during $0.1-\mathrm{Hz}$ stimulation at PT $(7.4 \pm 1.3 \mathrm{~min}, \mathrm{n}=8 ; \mathrm{p}=0.003$, paired $t$ test), we utilized the initial slope of recovery to estimate the recovery rate after GABA uncaging (Figure $3 \mathrm{~A}$ ). We also measured the percentage of recovery of IPSCs from the peak after uncaging (Figure $3 \mathrm{~A}$ ). Because efficiency of GABA uncaging is variable and the amount of photo-released GABA is unpredictable (Trigo et al., 2009), we utilized DPNI-GABA at a fixed concentration $(10 \mathrm{mM})$ and sampled data showing different initial slopes and peaks of recovery (Figure $3 \mathrm{C}$ ). We then compared recovery percentages of IPSCs after GABA uncaging with those obtained by infusion of GABA of defined concentrations (Figures 1C-1G). This enabled us to estimate GABA concentrations after GABA uncaging (Figure 3D), as previously estimated for glutamate concentrations after uncaging at the calyx of Held (Hori 
and Takahashi, 2012). Lineweaver-Burk plots of these data (Figure $3 \mathrm{E}$ ) gave estimates of $40 \mathrm{~s}$ for the time constant of maximal GABA uptake rate and $5.0 \mathrm{mM}$ for $\mathrm{K}_{\mathrm{M}}$ at PT. Corresponding estimates were $82 \mathrm{~s}$ and $3.1 \mathrm{mM}$ at RT (data not shown), indicating a temperature coefficient $\left(Q_{10}\right)$ of 2.5 for vesicular $G A B A$ uptake rate. This temperature coefficient coincided with that of the vesicular glutamate uptake rate $\left(Q_{10}=2.5\right.$; Hori and Takahashi, 2012). Thus, the vesicular GABA uptake rate was 5-6 times slower than that of glutamate uptake estimated at the calyx of Held (7 s at PT and $15 \mathrm{~s}$ at RT; Hori and Takahashi, 2012). The $K_{M}$ of GABA uptake estimated here at BC-PC synapses also coincided with that reported for the vesicular GABA transporter (VGAT) reconstituted in PC12 cells $(5 \mathrm{mM}$; Mclntire et al., 1997).

\section{Impact of Slow GABA Uptake on the Recovery of} Inhibitory Neurotransmission from STD

Given that vesicular GABA uptake is slow, it might limit IPSC recovery from STD. To examine this possibility, we induced STD of IPSCs using a 2-min train of stimulation at $50 \mathrm{~Hz}$ (Figure 4), which depressed IPSCs by $77.8 \pm 4.0 \%(n=5)$, suggesting that SVs in $\mathrm{BC}$ terminals were largely depleted. We first measured recovery of IPSCs from STD induced by a $50-\mathrm{Hz}$ train at intact synapses, without presynaptic perturbation by pipette solution (Figure 4A). At these synapses, IPSCs recovered with a time constant of $55 \pm 21 \mathrm{~s}$ at PT $(\mathrm{n}=5$; Figures $4 \mathrm{~A}$ and $4 \mathrm{~F})$. We next loaded GABA at different concentrations (1-10 mM) into BCs and measured the recovery of IPSCs from STD (Figures 4B-4E). Recovery was faster when the cytosolic GABA concentration was higher, with significant differences between time constants at different GABA concentrations (Figure 4F). The recovery time constants showed an inverse correlation with GABA concentrations $(r=0.99)$, with a correlation coefficient close to that of vesicular uptake time constant at different GABA concentrations $(r=0.93$; Figures $3 \mathrm{D}$ and $4 \mathrm{G} ; \mathrm{p}=0.32$ ). These results strongly suggest that vesicular GABA uptake limits recovery of IPSCs from STD induced by a $50-\mathrm{Hz}$ stimulation train.

In the cerebellar cortex, GABAergic inhibitory neurons fire spontaneously in bursts, at tens of hertz on average, thereby tonically inhibiting postsynaptic cells (Barmack and Yakhnitsa, 2008; Ruigrok et al., 2011; Häusser and Clark, 1997). Since the firing frequency of $\mathrm{BCs}$ in the molecular layer is reportedly 5-12 Hz, we measured the recovery of IPSCs from STD induced by $10-\mathrm{Hz}$ stimulation (Figures $4 \mathrm{~B}-4 \mathrm{G}$ ). At synapses where $\mathrm{BCs}$ were loaded with $5 \mathrm{mM}$ GABA, a 2-min train at $10 \mathrm{~Hz}$ depressed IPSCs by $52.3 \pm 3.9 \%(n=6)$. This STD magnitude was significantly less than after $50-\mathrm{Hz}$ train stimulation $(97.3 \pm 2.1 \%$, $\mathrm{n}=7$; Student's t test, $\mathrm{p}=0.0000087$; Figure 4D), suggesting less depletion of SVs from BC terminals. After $10-\mathrm{Hz}$ stimulation, recovery of IPSCs took bi-exponential time course with fast and slow time constants (Figures 4B-4E). The time constant of $3 \mathrm{~s}$ was judged best fit, by the least-squares method, to the fast recovery component after $10-\mathrm{Hz}$ stimulation, at all GABA concentrations used (1-10 mM). The proportion of the fast component was $19 \%-31 \%$. The slow time constants of recovery showed a clear dependency upon GABA concentrations ( $r=0.97)$, like vesicular GABA uptake time constant (Figure 3E; $p=0.69$ ). These results suggest that vesicular GABA uptake can serve as a rate-limiting factor for the recovery of IPSCs from STD in physiological conditions.

\section{DISCUSSION}

The refilling of vesicles with neurotransmitter is an essential step in SV recycling for maintaining the efficacy of neurotransmission. The time required for SV refilling is determined for glutamate at the excitatory calyx of Held synapse (Hori and Takahashi, 2012) but remained unknown at inhibitory synapses. Using GABA uncaging at BC-PC inhibitory synaptic pairs in cerebellar slices of juvenile rats, we have determined the time constant of GABA uptake into SVs as $40 \mathrm{~s}$ at PT. This time constant is 5-6 times slower than that of vesicular glutamate uptake (Hori and Takahashi, 2012). In both glutamatergic and GABAergic synapses, uptake of neurotransmitter by SVs is driven by a proton gradient generated by the vacuolar ATPase. Glutamate uptake by vesicular glutamate transporters (VGLUTs) depends mainly upon the trans-vesicular voltage difference in electrochemical equilibrium with proton concentration gradient produced by vacuolar ATPase (Blakely and Edwards, 2012), whereas vesicular GABA uptake by VGATs is mediated by an $\mathrm{H}^{+}$/GABA anti-port (Farsi et al., 2016) following vesicle acidification (Egashira et al., 2016). Our results indicate that the latter uptake mechanism takes a much longer time to refill vesicles with neurotransmitter than the former mechanism. Using a $\mathrm{pH}$ indicator, mOrange, in hippocampal cell culture, Egashira et al. (2016) estimated the GABAergic vesicle re-alkalization time constant as $25 \mathrm{~s}$ at PT. This time constant is faster than that of GABA uptake into SVs estimated at inhibitory synapses in this study.

The slow uptake rate of GABA into vesicles predicts that recycling reuse of SVs can be limited by the refilling rate unless there are enough SVs in terminals already filled. Filled SVs can be depleted, at least partially, during bursts of presynaptic firing at cerebellar inhibitory neurons (Barmack and Yakhnitsa, 2008; Ruigrok et al., 2011; Häusser and Clark, 1997). In our experimental conditions, SVs in BC terminals were strongly depleted during presynaptic stimulation at $50 \mathrm{~Hz}$ and more mildly during $10 \mathrm{~Hz}$ stimulation. In both cases, presynaptic GABA concentrations affected the time constant of IPSC recovery from STD, as they do for the vesicular GABA uptake time constant. The time constants of IPSC recovery after $50-\mathrm{Hz}$ stimulation and slow recovery time constants after $10-\mathrm{Hz}$ stimulation showed inverse relationships with presynaptic GABA concentrations, with a slope similar to that between GABA concentrations and vesicular GABA uptake time constants. Recovery of synaptic responses from STD can be accelerated if postsynaptic receptors are saturated with vesicular neurotransmitter (Foster et al., 2002). However, our results from presynaptic GABA infusion indicate a significant potentiation of IPSC amplitude above baseline after 10-mM GABA infusion (Figure 1G; $p=0.033$ ), suggesting that postsynaptic GABA receptors are not saturated with vesicular GABA at normal or lower GABA concentrations.

At excitatory synapses, multiple mechanisms are involved in STD (Zucker and Regehr, 2002; Schneggenburger et al., 2002), including depletion of releasable vesicles (Betz, 1970), reduction in the number of available release sites (Neher, 

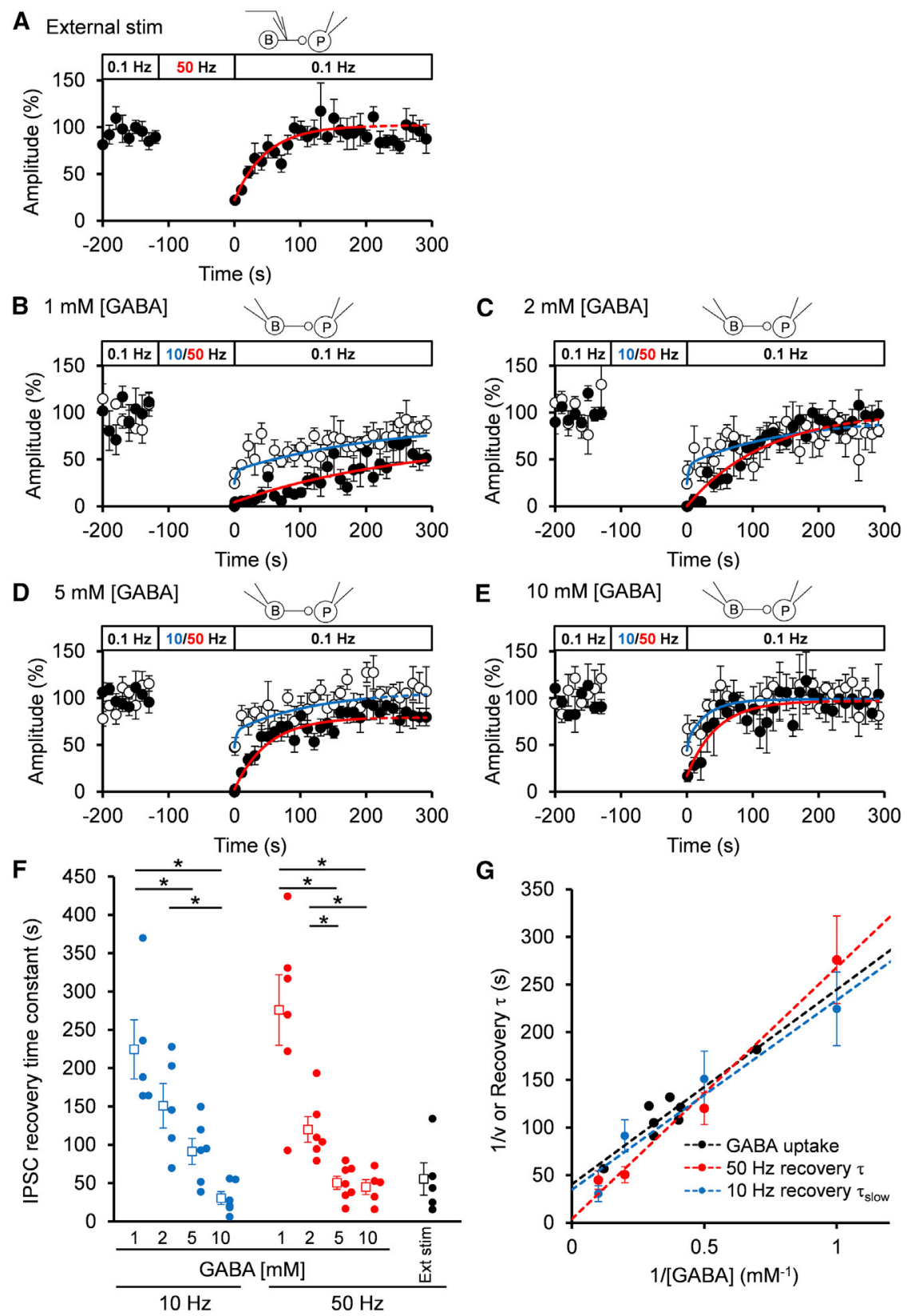

Figure 4. Recovery Rate of IPSCs from STD Depends upon Cytosolic GABA Concentration

(A-E) Time course of the recovery of IPSC amplitude during low-frequency stimulation $(0.1 \mathrm{~Hz})$ after STD (data not shown) induced by a train of highfrequency stimulation $(10 \mathrm{~Hz} \times 1,200$ or $50 \mathrm{~Hz} \times$ $6,000)$. BCs were stimulated at $0.1 \mathrm{~Hz}$ and switched to $10 \mathrm{~Hz}$ or $50 \mathrm{~Hz}$. Thereafter, stimulation frequency was returned to $0.1 \mathrm{~Hz}$. Data points, up to $300 \mathrm{~s}$ for $1 \mathrm{mM}$ [GABA] and $200 \mathrm{~s}$ for other conditions after switching to $0.1-\mathrm{Hz}$ stimulation, were fit by regression lines (red lines indicate $50 \mathrm{~Hz}$, and blue lines indicate $10 \mathrm{~Hz}$ ) using the least-squares method. Experiments were performed at PT. B, presynaptic $\mathrm{BC} ; \mathrm{P}$, postsynaptic $\mathrm{PC}$

(A) IPSCs were evoked by extracellular stimulation without whole-cell recording from $\mathrm{BCs}$.

(B-E) IPSCs were evoked by action potentials generated in BCs in the presence of GABA at $1 \mathrm{mM}$ (B), $2 \mathrm{mM}(\mathrm{C}), 5 \mathrm{mM}(\mathrm{D})$, and $10 \mathrm{mM}(\mathrm{E})$. Recovery after $10-\mathrm{Hz}$ stimulation was best fit with bi-exponential curves (blue) with a fast time constant of $3 \mathrm{~s}$ and a slow time constant of 31-225 s, depending on cytosolic GABA concentrations. The proportions of the fast component were $21 \%, 31 \%$, $29 \%$, and $19 \%$, at GABA concentrations of $1 \mathrm{mM}$, $2 \mathrm{mM}, 5 \mathrm{mM}$, and $10 \mathrm{mM}$, respectively.

(F) Recovery time constants of IPSCs from STD (blue circles, $10 \mathrm{~Hz}$; red circles, $50 \mathrm{~Hz}$ ) without a presynaptic whole-cell recording (filled black circles; $n=5$ ) or in a whole-cell recording from BCs with different GABA concentrations $(n=5$ at $10 \mathrm{mM}$ [GABA], $\mathrm{n}=6$ at $1 \mathrm{mM}$ and $2 \mathrm{mM}$ [GABA], and $\mathrm{n}=7$ at $5 \mathrm{mM}[\mathrm{GABA}]$ at $50 \mathrm{~Hz} ; \mathrm{n}=5$ at $1 \mathrm{mM}$ and $2 \mathrm{mM}$ [GABA], and $\mathrm{n}=6$ at $5 \mathrm{mM}$ and $10 \mathrm{mM}$ [GABA] at $10 \mathrm{~Hz}$ ). The recovery time constants after STD were statistically significant (non-parametric Steel-Dwass test) between $1 \mathrm{mM}$ and $5 \mathrm{mM}$ $(p=0.02)$, between $1 \mathrm{mM}$ and $10 \mathrm{mM}(p=0.04)$, between $2 \mathrm{mM}$ and $5 \mathrm{mM}(\mathrm{p}=0.03)$, and between $2 \mathrm{mM}$ and $10 \mathrm{mM}(p=0.04)$ at $50 \mathrm{~Hz}$. At $10 \mathrm{~Hz}$, the slow recovery time constants are statistically significant between $1 \mathrm{mM}$ and $5 \mathrm{mM}(\mathrm{p}=0.04)$, between $1 \mathrm{mM}$ and $10 \mathrm{mM}(\mathrm{p}=0.04)$, and between $2 \mathrm{mM}$ and $10 \mathrm{mM}(\mathrm{p}=0.04)$. Asterisks indicate statistical significance $(p<0.05)$.

(G) 1/v (ordinate) of vesicular GABA uptake (filled black circles; data are taken from Figure $3 \mathrm{E}$ ) and recovery time constants $(\tau)$ from STD (red circles, $50 \mathrm{~Hz}$; blue circles, $10 \mathrm{~Hz}$; slow time constant; data are taken from Figure 4F) at different presynaptic cytosolic GABA concentrations (abscissa). Correlation coefficients: $r=0.93$ for GABA uptake; rs $=0.99$ and 0.97 for IPSC recovery after STD at $50 \mathrm{~Hz}$ and $10 \mathrm{~Hz}$, respectively. There was no significant difference between the correlation coefficients between recovery from $50-\mathrm{Hz}$ STD and GABA uptake $(p=0.32)$ as well as between recovery from 10-Hz STD and GABA uptake $(p=0.69)$.

Values are given as mean \pm SEM.

2010), inactivation of presynaptic $\mathrm{Ca}^{2+}$ channels (Forsythe et al., 1998), or desensitization of postsynaptic receptors (Saviane and Silver, 2006). At calyx of Held synapses, recovery from STD has fast and slow components, with the former being dependent on $\mathrm{Ca}^{2+}$ (Wang and Kaczmarek, 1998) and calmodulin (Sakaba and Neher, 2001), whereas the latter reflects GTP-dependent SV replenishment (Takahashi et al., 2000) or recovery of presynaptic $\mathrm{Ca}^{2+}$ channels from inactivation (Forsythe et al., 1998). Whereas much less is known about the mechanisms underlying the recov- ery of inhibitory neurotransmission from STD, our present results indicate that vesicular GABA uptake rate can limit the recovery rate of inhibitory neurotransmission from STD.

Since presynaptic cell firing activity varies from time to time and among different synapses, the magnitude of SV depletion from presynaptic terminals may also vary. When SV depletion is mild, recovery of IPSCs from depression is produced by a replenishment of release sites with already filled SVs, together with newly recycled and refilled SVs. In such cases, 
the contribution of the refilling rate to the IPSC recovery rate is expectedly partial. In fact, after $10-\mathrm{Hz}$ stimulation, recovery of IPSCs had a bi-exponential time course (Figures 4B-4E). The fast recovery time constant was much faster than GABA uptake time constant and showed no GABA-concentration dependence. In contrast, the slow time constant was similar to the vesicular GABA uptake time constant and, likewise, correlated with presynaptic cytosolic GABA concentrations (Figures 4F and 4G). At hippocampal inhibitory synapses, the recovery of IPSCs from STD, induced by a train of $20 \mathrm{~Hz} \times 50$-s stimulation, reportedly follows a bi-exponential time course with time constants of $1.3 \mathrm{~s}$ and $32 \mathrm{~s}$ estimated at PT (Kraushaar and Jonas, 2000). This slow recovery time constant is comparable to that at $\mathrm{BC}$ $\mathrm{PC}$ synapses, suggesting that it might be determined by vesicular GABA uptake rate at hippocampal inhibitory synapses. Like GABA, vesicular glycine uptake is mediated by VGAT (Mclntire et al., 1997). Thus, the vesicular refilling rate of glycine may also be slow and may affect the recovery rate of glycinergic inhibitory transmission from STD.

The expression level of vesicular transporter is correlated with the rate of transmitter uptake (Wilson et al., 2005). The present study was made at BC-PC synapses in rat cerebellum at postnatal day (P)12-P16. At this age, expression levels of both VGAT (Minelli et al., 2003) and VGLUT (Billups, 2005; Blaesse et al., 2005), as well as the vesicular glutamate uptake rate (Hori and Takahashi, 2012), reach a plateau, suggesting that vesicular GABA uptake rate measured here probably persists into adult ages. However, the question remains open as to what extent the GABA uptake rate limits the recovery of IPSCs from STD in adult animals.

In the presence of both excitatory and inhibitory synaptic inputs, postsynaptic cell firing tends to be enhanced during STD of inhibitory transmission. In the cerebellar cortical molecular layer, BCs and stellate cells provide significant inhibitory drives to PCs (Andersen et al., 1963), mediating feed-forward inhibition from parallel fibers to PCs. BCs also provide tonic inhibition on PCs by firing spontaneously at a high frequency up to tens of hertz (Eccles et al., 1966; Häusser and Clark, 1997; Ruigrok et al., 2011). Thus, the slow recovery of IPSCs from STD, due to slow vesicular GABA uptake rate, may disinhibit PCs for a sustained period, thereby promoting the high excitability of PCs.

\section{EXPERIMENTAL PROCEDURES}

All experiments were performed in accordance with the guidelines of Doshisha University and the Physiological Society of Japan.

\section{Slice Preparation}

After decapitation, sagittal slices $(200 \mu \mathrm{m}$ thick) were cut from the cerebellar cortex of Wistar/ST rats (P 12-16) of either sex. Slices were superfused with artificial cerebrospinal fluid (aCSF) containing (in millimolar): $125 \mathrm{NaCl}$, $2.5 \mathrm{KCl}, 26 \mathrm{NaH}_{2} \mathrm{PO}_{4}, 2 \mathrm{CaCl}_{2}, 6 \mathrm{MgCl}_{2}, 10$ glucose, 3 myo-inositol, 2 sodium pyruvate, and 5 ascorbic acid $(\mathrm{pH} 7.3$, when bubbled with $95 \%$ $\mathrm{O}_{2} / 5 \% \mathrm{CO}_{2}$ ).

\section{Culture}

Primary cultures of cerebellar neurons were prepared from newborn Wistar rats of both sexes, as previously reported (Kawaguchi and Sakaba, 2015). For details, see the Supplemental Experimental Procedures.
Electrophysiology and DPNI-GABA Uncaging

Electrophysiological experiments were performed as in previous studies (Hori and Takahashi, 2012; Kawaguchi and Sakaba, 2015). Detailed information is available in the Supplemental Experimental Procedures.

\section{Data Analysis}

Data fitting was performed using the least-squares method (single or double exponential). All values are given as mean $\pm \mathrm{SEM}$; $n$ represents number of experiments. A difference of $p<0.05$, in Student's t test, paired t test, repeatedmeasures ANOVA, comparison of two correlation coefficients, or Steel-Dwass test, was regarded as significant by Excel 2013 or JMP Pro 13.

\section{SUPPLEMENTAL INFORMATION}

Supplemental Information includes Supplemental Experimental Procedures and two figures and can be found with this article online at https://doi.org/ 10.1016/j.celrep.2018.02.080.

\section{ACKNOWLEDGMENTS}

This work was supported by the JSPS Core-to-Core Program A, Advanced Research Networks, and by the Okinawa Institute of Science and Technology (OIST). We thank Alain Marty and Federico Trigo for discussions and technical advice, Takeshi Sakaba and Shigeo Takamori for comments, and Steven D. Aird for editing the manuscript.

\section{AUTHOR CONTRIBUTIONS}

M.Y., T.H., and T.T. designed experiments; M.Y., S.K., and T.H. performed experiments and data analysis; and M.Y., T.H., and T.T. wrote the paper.

\section{DECLARATION OF INTERESTS}

The authors declare no competing interests.

Received: October 15, 2016

Revised: January 5, 2018

Accepted: February 21, 2018

Published: March 20, 2018

\section{REFERENCES}

Andersen, P., Eccles, J., and Voorhoeve, P.E. (1963). Inhibitory synapses on somas of Purkinje cells in the cerebellum. Nature 199, 655-656.

Apostolides, P.F., and Trussell, L.O. (2013). Rapid, activity-independent turnover of vesicular transmitter content at a mixed glycine/GABA synapse. J. Neurosci. 33, 4768-4781.

Barmack, N.H., and Yakhnitsa, V. (2008). Functions of interneurons in mouse cerebellum. J. Neurosci. 28, 1140-1152.

Betz, W.J. (1970). Depression of transmitter release at the neuromuscular junction of the frog. J. Physiol. 206, 629-644.

Billups, B. (2005). Colocalization of vesicular glutamate transporters in the rat superior olivary complex. Neurosci. Lett. 382, 66-70.

Blaesse, P., Ehrhardt, S., Friauf, E., and Nothwang, H.G. (2005). Developmental pattern of three vesicular glutamate transporters in the rat superior olivary complex. Cell Tissue Res. 320, 33-50.

Blakely, R.D., and Edwards, R.H. (2012). Vesicular and plasma membrane transporters for neurotransmitters. Cold Spring Harb. Perspect. Biol. 4, a005595.

Carlson, M.D., Kish, P.E., and Ueda, T. (1989). Characterization of the solubilized and reconstituted ATP-dependent vesicular glutamate uptake system. J. Biol. Chem. 264, 7369-7376.

Eccles, J.C., Llinás, R., and Sasaki, K. (1966). The inhibitory interneurones within the cerebellar cortex. Exp. Brain Res. 1, 1-16. 
Egashira, Y., Takase, M., Watanabe, S., Ishida, J., Fukamizu, A., Kaneko, R., Yanagawa, Y., and Takamori, S. (2016). Unique pH dynamics in GABAergic synaptic vesicles illuminates the mechanism and kinetics of GABA loading. Proc. Natl. Acad. Sci. USA 113, 10702-10707.

Farsi, Z., Preobraschenski, J., van den Bogaart, G., Riedel, D., Jahn, R., and Woehler, A. (2016). Single-vesicle imaging reveals different transport mechanisms between glutamatergic and GABAergic vesicles. Science 351, 981-984.

Forsythe, I.D., Tsujimoto, T., Barnes-Davies, M., Cuttle, M.F., and Takahashi, T. (1998). Inactivation of presynaptic calcium current contributes to synaptic depression at a fast central synapse. Neuron 20, 797-807.

Foster, K.A., Kreitzer, A.C., and Regehr, W.G. (2002). Interaction of postsynaptic receptor saturation with presynaptic mechanisms produces a reliable synapse. Neuron 36, 1115-1126.

Häusser, M., and Clark, B.A. (1997). Tonic synaptic inhibition modulates neuronal output pattern and spatiotemporal synaptic integration. Neuron 19, 665-678.

Hell, J.W., Maycox, P.R., and Jahn, R. (1990). Energy dependence and functional reconstitution of the $\gamma$-aminobutyric acid carrier from synaptic vesicles. J. Biol. Chem. 265, 2111-2117.

Heuser, J.E., and Reese, T.S. (1973). Evidence for recycling of synaptic vesicle membrane during transmitter release at the frog neuromuscular junction. J. Cell Biol. 57, 315-344.

Hori, T., and Takahashi, T. (2012). Kinetics of synaptic vesicle refilling with neurotransmitter glutamate. Neuron 76, 511-517.

Hori, T., Takai, Y., and Takahashi, T. (1999). Presynaptic mechanism for phorbol ester-induced synaptic potentiation. J. Neurosci. 19, 7262-7267.

Ishikawa, T., Sahara, Y., and Takahashi, T. (2002). A single packet of transmitter does not saturate postsynaptic glutamate receptors. Neuron 34, 613-621.

Kawaguchi, S.Y., and Sakaba, T. (2015). Control of inhibitory synaptic outputs by low excitability of axon terminals revealed by direct recording. Neuron 85 , 1273-1288.

Kish, P.E., Fischer-Bovenkerk, C., and Ueda, T. (1989). Active transport of $\gamma$-aminobutyric acid and glycine into synaptic vesicles. Proc. Natl. Acad. Sci. USA 86, 3877-3881.

Kraushaar, U., and Jonas, P. (2000). Efficacy and stability of quantal GABA release at a hippocampal interneuron-principal neuron synapse. J. Neurosci. 20, 5594-5607.

Liu, G., and Tsien, R.W. (1995). Properties of synaptic transmission at single hippocampal synaptic boutons. Nature 375, 404-408.

Maycox, P.R., Deckwerth, T., Hell, J.W., and Jahn, R. (1988). Glutamate uptake by brain synaptic vesicles. Energy dependence of transport and functional reconstitution in proteoliposomes. J. Biol. Chem. 263, 15423-15428.

McIntire, S.L., Reimer, R.J., Schuske, K., Edwards, R.H., and Jorgensen, E.M. (1997). Identification and characterization of the vesicular GABA transporter. Nature 389, 870-876.
Minelli, A., Alonso-Nanclares, L., Edwards, R.H., DeFelipe, J., and Conti, F. (2003). Postnatal development of the vesicular GABA transporter in rat cerebral cortex. Neuroscience 117, 337-346.

Neher, E. (2010). What is rate-limiting during sustained synaptic activity: vesicle supply or the availability of release sites. Front. Synaptic Neurosci. 2, 144.

Otsuka, M., Obata, K., Miyata, Y., and Tanaka, Y. (1971). Measurement of $\gamma$-aminobutyric acid in isolated nerve cells of cat central nervous system. J. Neurochem. 18, 287-295.

Rizzoli, S.O. (2014). Synaptic vesicle recycling: steps and principles. EMBO J. 33, 788-822.

Ruigrok, T.J., Hensbroek, R.A., and Simpson, J.I. (2011). Spontaneous activity signatures of morphologically identified interneurons in the vestibulocerebellum. J. Neurosci. 31, 712-724.

Ryan, T.A., Reuter, H., Wendland, B., Schweizer, F.E., Tsien, R.W., and Smith, S.J. (1993). The kinetics of synaptic vesicle recycling measured at single presynaptic boutons. Neuron 11, 713-724.

Sakaba, T., and Neher, E. (2001). Calmodulin mediates rapid recruitment of fast-releasing synaptic vesicles at a calyx-type synapse. Neuron 32, 11191131.

Saviane, C., and Silver, R.A. (2006). Fast vesicle reloading and a large pool sustain high bandwidth transmission at a central synapse. Nature 439, 983-987.

Schneggenburger, R., Sakaba, T., and Neher, E. (2002). Vesicle pools and short-term synaptic depression: lessons from a large synapse. Trends Neurosci. 25, 206-212.

Takahashi, T., Hori, T., Kajikawa, Y., and Tsujimoto, T. (2000). The role of GTP binding protein activity in fast central synaptic transmission. Science 289, 460-463.

Takami, C., Eguchi, K., Hori, T., and Takahashi, T. (2017). Impact of vesicular glutamate leakage on synaptic transmission at the calyx of Held. J. Physiol. 595, 1263-1271.

Trigo, F.F., Papageorgiou, G., Corrie, J.E.T., and Ogden, D. (2009). Laser photolysis of DPNI-GABA, a tool for investigating the properties and distribution of GABA receptors and for silencing neurons in situ. J. Neurosci. Methods $181,159-169$.

Wang, L.Y., and Kaczmarek, L.K. (1998). High-frequency firing helps replenish the readily releasable pool of synaptic vesicles. Nature 394, 384-388.

Wang, L., Tu, P., Bonet, L., Aubrey, K.R., and Supplisson, S. (2013). Cytosolic transmitter concentration regulates vesicle cycling at hippocampal GABAergic terminals. Neuron 80, 143-158.

Wilson, N.R., Kang, J., Hueske, E.V., Leung, T., Varoqui, H., Murnick, J.G., Erickson, J.D., and Liu, G. (2005). Presynaptic regulation of quantal size by the vesicular glutamate transporter VGLUT1. J. Neurosci. 25, 6221-6234.

Yamashita, T., Kanda, T., Eguchi, K., and Takahashi, T. (2009). Vesicular glutamate filling and AMPA receptor occupancy at the calyx of Held synapse of immature rats. J. Physiol. 587, 2327-2339.

Zucker, R.S., and Regehr, W.G. (2002). Short-term synaptic plasticity. Annu. Rev. Physiol. 64, 355-405. 